\title{
LifeFirst: Impact of a school-based tobacco and supari cessation intervention among adolescent students in Mumbai, India
}

\author{
Upendra Bhojani ${ }^{1}$, Amiti Varma ${ }^{1}$, Pragati B. Hebbar ${ }^{1}$, Gauri Mandal ${ }^{2}$, Himanshu Gupte ${ }^{3}$
}

\section{AFFILIATION}

1 Cluster on Chronic Health Conditions \& Public Policy, Institute of Public Health, Bengaluru, India

2 Salaam Bombay Foundation, Mumbai, India

3 Narotam Sekhsaria Foundation, Mumbai, India

CORRESPONDENCE TO

Upendra Bhojani. Institute of Public Health, 3009, II-A Main, 17th Cross,
Krishna Rajendra Rd, Banashankari Stage II, Bengaluru, Karnataka, 560070, India.E-mail: upendra@iphindia.org

\section{KEYWORDS}

school-based intervention, youth, tobacco, supari, cessation, India

Received: 28 August 2020, Revised: 18 January 2021,

Accepted: 25 March 2021

Popul. Med. 2021;3(May):12

https://doi.org/10.18332/popmed/134990

\begin{abstract}
INTRODUCTION The youth are a vulnerable populationgroup for tobacco-related harms. Schools are an excellent setting for health promotion; yet there is a dearth of schoolbased cessation interventions, rarely evaluated for their impact. Here, we assess the impact of the LifeFirst program: an ongoing tobacco and supari (areca nut) cessation intervention delivered to students from corporation schools in Mumbai city.

METHODS We used a prospective quasi-experimental design with an intervention and a control arm embedded within an ongoing LifeFirst program in select schools. We used a difference-in-difference analysis with baseline and endline surveys to assess the program's impact on students' knowledge about harms, students' refusal skills, and prevalence of tobacco/supari use. We report our work using the TREND statement checklist.
\end{abstract}

RESULTS A total of 959 students registered in the LifeFirst program. In our analysis, we included 827 students who completed both the baseline and end-line surveys. Postintervention, we found both tobacco and supari use reduced substantially among the intervention group while tobacco use increased among the control group. The difference-indifference estimates show a statistically significant reduction of 17.9 and 38.1 percentage points in the intervention group for tobacco and supari use respectively, beyond the reduction in the control group.

CONCLUSIONS The LifeFirst program was successful in reducing tobacco and supari use among the study participants and protected students in the intervention group against new uptake of tobacco. It helped improve knowledge score and refusal skills among students. Implementation and evaluation of similar school-based programs should be considered as part of a multi-strategy approach to reducing tobacco use among young people.

\section{INTRODUCTION}

India has the second largest number of tobacco users in the world ${ }^{1}$. Tobacco use accounts for over 1.3 million deaths a year in the country ${ }^{2,3}$ and the youth remain among the most vulnerable population segments in terms of tobacco-related harms. It is at this stage in life that people usually experiment and/or initiate tobacco use. In India, the average age at which people take up tobacco use is 18.9 years ${ }^{4}$. Tobacco use among youth in India is quite high: $14.6 \%$ users in the age group $13-15$ years in the year $2009^{5}$, and $12.4 \%$ users in the age group 15-24 years in the years 2016-20176. Tobacco is also used in diverse forms. Analysis of a nationally representative survey done in 2009 revealed that among youth tobacco users, $35.1 \%$ smoked cigarettes or bidis and $43.3 \%$ used chewing or smokeless tobacco, while $21.5 \%$ youth used more than one form of tobacco ${ }^{7}$.

Along with tobacco use, use of supari (areca nut) in various forms, included as an ingredient in smokeless tobacco products, is common in India and is linked to various health hazards including oral cancers ${ }^{8}$. Studies among youth in Mumbai (India) report the prevalence of ever use of supari products to be from about $18 \%$ to about $32 \% 0^{9,10}$. Adding to 
their vulnerability, studies have shown that the tobacco industry specifically targets youth through their marketing strategies ${ }^{11}$. Studies have shown an association between youth uptake of tobacco use and having parents, family members and peers using tobacco, exposure to tobacco portrayal in media, undergoing stressful episodes, and access to disposable pocket money ${ }^{12,13}$.

Schools provide an excellent setting for health promotion interventions in engaging youth and related stakeholders ${ }^{14}$. While there have been regulatory measures (e.g. ban on sale of tobacco to minors and around educational institutions ${ }^{15}$, and educational interventions such as school-based programs as part of the National Tobacco Control Program ${ }^{16}$ ), there is a dearth of school-based interventions offering support to youth to quit tobacco. This is despite a huge need: nearly two-thirds of the student smokers expressed desire to quit smoking in a national survey ${ }^{5}$. In fact, offering cessation support to current users is recognized as an important strategy by the World Health Organization in its MPOWER strategy package for tobacco control ${ }^{17}$. While documented interventions in this domain in India are themselves limited in number, they are rarely evaluated for their impact. At present, there is limited evidence available in the form of two different interventions, one for school teachers in Bihar (2009-2011) ${ }^{18}$ and another for school students (6th and 8th grades) from Delhi and Chennai cities (2004-2006) ${ }^{19}$, which provided positive evidence for potential of schoolbased tobacco control interventions in reducing tobacco use. Furthermore, systematic reviews of the studies around smoking or tobacco cessation have pointed to the positive impact of group-based counselling sessions ${ }^{20,21}$.

It is in this context of very limited evidence around school-based tobacco cessation interventions that we aimed to assess the impact of the LifeFirst program. LifeFirst is an ongoing tobacco and supari cessation intervention delivered to the students from low socioeconomic stratum studying in corporation (local government) schools in Mumbai city ${ }^{22}$. The specific objectives of our study conducted in intervention and control schools in Mumbai were to assess and compare the change in: 1) prevalence of tobacco and supari use, 2) knowledge about harms of tobacco and supari, and 3) perceived skills to refuse tobacco and supari among the students receiving and not receiving the LifeFirst tobacco and supari cessation intervention in school.

\section{METHODS}

\section{Study design}

We used a prospective quasi-experimental design with an intervention and a control arm embedded within an ongoing LifeFirst tobacco and supari cessation program. The participants of the program were students who wished to quit their tobacco and supari usage. The students assigned to the intervention group/schools registered and received LifeFirst program intervention while those assigned to the control group/schools were registered for the LifeFirst program but were to receive the program intervention in the subsequent academic year. Hence, the latter group served as a natural control. A Transparent Reporting of Evaluations with Nonrandomized Designs (TREND) statement checklist provides the details of the various study components and their location in the manuscript (See Supplementary File) ${ }^{23}$.

We used a difference-in-difference analysis ${ }^{24}$ with baseline and end-line questionnaires to assess the program's impact on students' knowledge about tobacco/supari harms, students' refusal skills with regard to tobacco/supari use, and the prevalence of tobacco/supari use. Figure 1 depicts the overall study design.

\section{About the LifeFirst intervention}

The LifeFirst is a standardized tobacco and supari cessation program conceived and implemented by the Narotam Sekhsaria Foundation, India, since 2012. The Program targets students from 7 th to 9 th grades in corporation (local government) funded/managed schools in Mumbai city. The typical program cycle runs through a given academic year and is delivered by trained program staff in the selected schools in a separate space allocated by the school authorities. It starts with an orientation program in select schools where students are sensitized about harms of tobacco and supari use. Following the orientation session, students using tobacco and/or supari are invited to register at their will (ie. voluntary registration) and be part of the main program, which includes delivery of six group-based education and counselling sessions by trained counsellors at a designated location within their respective schools. These interactive sessions are of 40-50 minutes duration conducted at monthly intervals and focus on specific themes (i.e. group bonding, tobacco harms, barriers, refusal skills). Intervention sessions focused on understanding the types of tobacco and supari products, associated health harms, ways of quitting, the concept of craving, temptation, withdrawal and coping mechanisms, and relapse. Additionally, as a part of refusal skills, the sessions emphasized on differentiating between passive, assertive and aggressive behaviors and the importance of assertive behavior and had activities that used roleplay of refusal skills for tobacco and supari products. In order to maintain attendance and participation, the program intervention was conducted at the school campus within school hours, and program activities were designed to be diverse and engaging. A more detailed description is provided in the Supplementary file. The program keeps record of students' participation and their tobacco/supari use status overtime.

\section{Sample size}

Our primary outcomes included the change in the proportion of tobacco users and supari users among registered students in the course of the program and comparing these measures across intervention and control groups. The closest relevant indicator we could use to estimate the required sample 


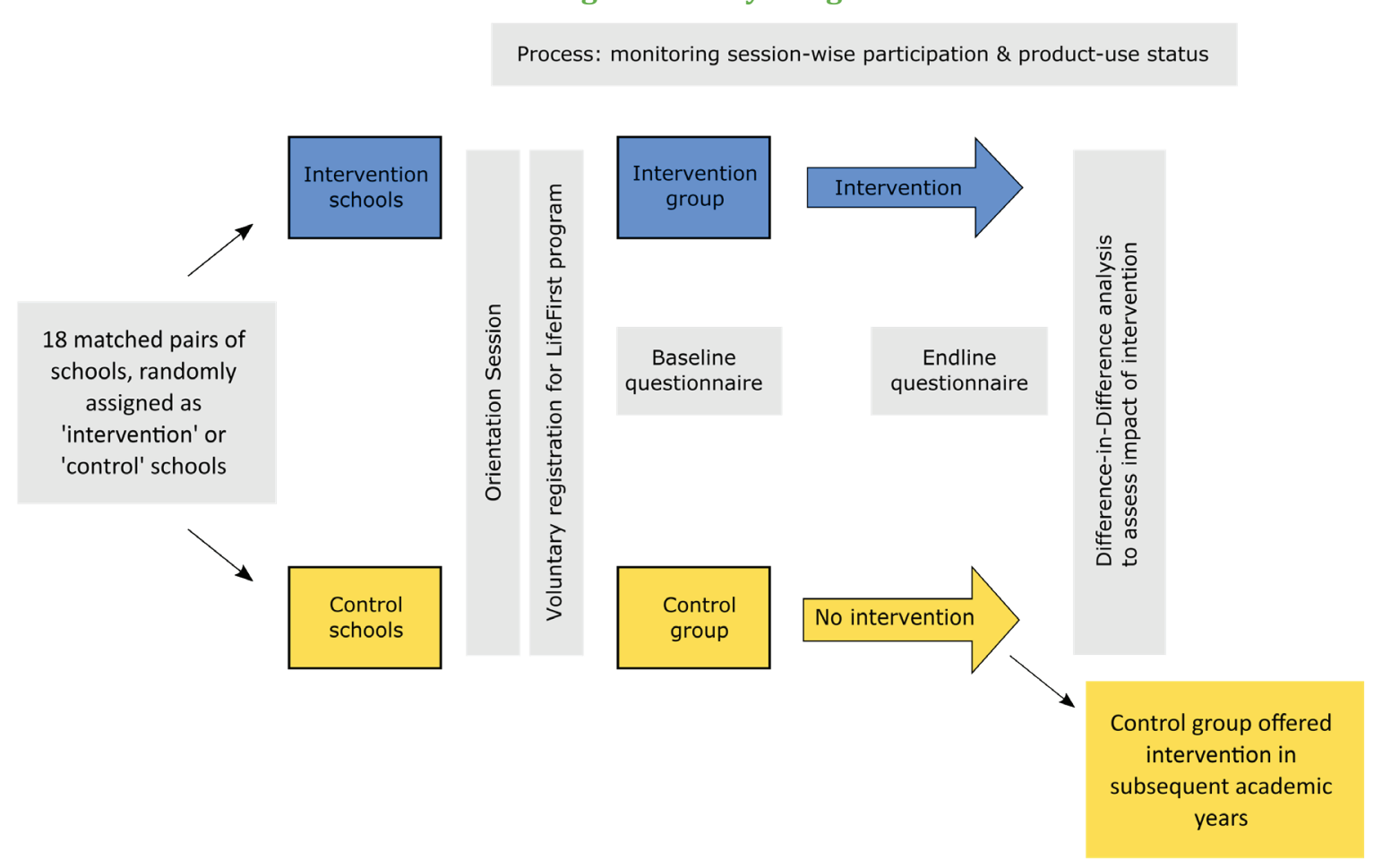

size for such a study came from the LifeFirst program data recorded for the earlier program cycles (2012-2013 to 2016-2017). On average, about $65 \%$ of registered students quit tobacco and/or supari use based on students' self-report at the end of the respective program cycles. While this was a mixed measure (for tobacco and supari) and not exactly the one we aimed to use for our study, we believe it provided a valuable guide to estimate the sample size for our study. Using this value, we used STATA to estimate the sample size required to detect an effect size of ten percentage points (a difference between intervention and control groups) with $80 \%$ power and significance level (alpha) of 0.05 . We added $10 \%$ to this number to compensate for any design defects arriving at a minimal sample size of about 436 students per group.

\section{Recruitment of participants}

Our study focused on the academic year June 2017 March 2018 and the associated program cycle of the LifeFirst. We first chose the schools and then recruited students from those schools into intervention and control groups. The Municipal Corporation of Greater Mumbai represents the largest primary education system in India: about 1162 schools (approximately 394599 students) directly funded and managed by the corporation (often referred as corporation schools) and about 450 schools (approximately 300504 students) receiving financial aid from the corporation but being managed privately (often referred to as aided schools) ${ }^{25}$. Corporation schools provide free education while the aided schools charge nominal fees, primarily catering to, and at times the only option for, economically weaker sections of Mumbai residents ${ }^{25}$. While these schools have been facing challenges of declining enrollment rates and suboptimal learning outcomes, they do provide seamless education from pre-primary up to 10th standards with low dropout rates ${ }^{26}$.

We matched the schools for which the LifeFirst had received necessary permissions to engage with in the given academic year on four criteria: their location (municipal ward), the medium of instruction (Hindi, Urdu, English, Marathi), the school type (corporation schools, aided schools), and the grades they offered (7th, 8th, 9th). We arrived at 18 matching pairs of schools. Within matched pairs, we randomly allocated one to the intervention group and the other to the control group. The LifeFirst staff, blinded to the allocation of schools into intervention and control groups, conducted orientation sessions on tobacco/supari harms in these schools and started enrolling the willing students (i.e. the current users of tobacco and/or supari who were willing to stop their use) into the LifeFirst program following these sessions in their respective schools. A real-time inventory was kept concerning the number of students being enrolled from intervention and control schools. We kept including schools till we reached the estimated sample size.

\section{Data collection}

We used a self-administered questionnaire to collect data from the students enrolled into the LifeFirst program. We 
administered the baseline questionnaire as a part of the first session of the LifeFirst program (before the intervention) and the end-line questionnaire in the last session of the program (after the intervention). The questionnaires used in these two rounds were identical, except for a section in the end-line questionnaire assessing the students' participation in and feedback on the LifeFirst program. The questionnaires broadly assessed: 1) personal and sociodemographic characteristics of students; 2) their knowledge, attitudes and practice concerning tobacco and supari use; 3) their quit attempts and outcomes; and 4) data on variables that are known to influence uptake of tobacco and supari (e.g. exposure to others using tobacco/ supari, portrayal of tobacco/supari use in media, access to tobacco/supari products, and knowledge and perceived enforcement of tobacco control policies in schools). The questionnaires adapted elements from tools that have been validated and used previously in India for assessing tobacco use and related factors among youth. These included the core questionnaire used in Global Youth Tobacco Survey (India) ${ }^{27}$ and a questionnaire used among pre-university students in Karnataka $^{28}$. The initial questionnaire developed in English was translated into Hindi, Marathi and Urdu, and was back translated into English to ensure accuracy in meaning.

LifeFirst staff, who undertook training for data collection for this study and were blinded to the intervention/control status of the schools, administered the questionnaires. The children participating in the study were also blinded to the intervention and control status and only the researchers evaluating the program were aware of the intervention and control group details. After securing consent, from the school authorities and parents of the children, data collectors provided instructions, to all the children in a separate space allocated by the school to conduct the LifeFirst related activities, distributed the questionnaires and assisted those students who had queries on any specific questions.

\section{Measures}

The primary outcome variables included point prevalence of tobacco use, and point prevalence of supari use, among students registered for LifeFirst program. The prevalence of tobacco and/or supari use was estimated based on the use of any products containing tobacco and/or supari in the 30 days preceding the questionnaire. The secondary outcome variables included knowledge score on tobacco harms and supari harms. These scores were arrived at by simple summation of scores for individual questions ( 1 for correct answer; 0 for incorrect answer) assessing knowledge on tobacco and supari harms. The tobacco knowledge score ranged from 0 to 6 , while the supari knowledge score ranged from 0 to 2 . We also included refusal scores for tobacco products among secondary outcomes, derived by simple summation of scores for individual questions (1 confirming refusal; 0 indicating non refusal) on students' ability to refuse offers of tobacco from others, and ranged from 0 to 3 .
We used a set of independent/exposure variables that are known to influence tobacco use uptake/status among youth. These included students' age, sex, school grade, medium of instruction, and pocket money.

\section{Statistical analysis}

Trained professionals generated electronic data from the baseline and end-line surveys using Epidata Manager $(\mathrm{v} \text { 4.6.0.0 })^{29}$. We used standard data entry forms with validity checks and manually verified $10 \%$ of the entered data using physical forms to ensure accuracy. We used Stata SE $15.1^{30}$ to analyze data. We conducted a difference-in-difference analysis for the five outcome variables in the study. The difference-in-difference analysis compares the changes in outcome variables over time between the intervention and control group, keeping in consideration the initial differences between these two groups. We also adjusted such analysis using relevant covariates. Finally, to promote transparency and wider use, we also published our dataset on Figshare, a publicly accessible research and data repository ${ }^{31}$.

\section{RESULTS}

A total of 959 students volunteered to register in the LifeFirst program in order to quit their tobacco/supari use habit. From this, 827 students completed both the baseline and end-line questionnaires, yielding a response rate of $86.2 \%$ and these students formed our population for analysis. The other 132 students (66 each in the intervention and control group) were not included as they answered either the baseline or the end-line survey. The reasons for nonresponse included absenteeism on the day of the surveys and/or transfer to other schools.

Table 1 provides characteristics of the final sample population including the outcome measures at the baseline, reported by study condition along with indication of statistically significant differences between the two groups. On the whole, $17 \%$ of students used tobacco and $97 \%$ used supari in the 30 days preceding the baseline survey. Most of the tobacco users used supari as well, with the proportion of dual users being $15.7 \%$ in the control group and $16.8 \%$ in the intervention group. We found a greater degree of participation by students in all the six thematic sessions in the intervention group: the median number of sessions participated by students in the intervention group was $5.07(\mathrm{SD}=1.2)$. Our recruitment strategy of using matched schools yielded a largely matching sample. Both the groups were identical in their background characteristics except for the educational level, medium of instruction, and exposure to media advertising.

In terms of outcomes at the baseline, the students in the control group had marginally higher knowledge scores for tobacco and supari harms. However, our use of the difference-in-difference analysis accounted for these initial differences across the two groups. Table 2 presents the findings from the difference-in-difference analysis for each of the outcome variables. 
Table 1. Major characteristics of the sample population at baseline

\begin{tabular}{|c|c|c|}
\hline & $\begin{array}{l}\text { Control } \\
(\mathrm{N}=414)\end{array}$ & $\begin{array}{l}\text { Intervention } \\
(\mathrm{N}=413)\end{array}$ \\
\hline \multicolumn{3}{|l|}{ Sex, n (\%) } \\
\hline Boys & $261(63.0)$ & $270(65.4)$ \\
\hline Girls & $153(37.0)$ & $143(34.6)$ \\
\hline Age (years), mean (SD) & $13.3(1.3)$ & $13.4(1.5)$ \\
\hline \multicolumn{3}{|l|}{ Current education level, $\mathrm{n}(\%)^{* *}$} \\
\hline 7 th grade & 107 (25.9) & $136(33.0)$ \\
\hline 8 th grade & $144(34.8)$ & $106(25.7)$ \\
\hline 9th grade & $163(39.4)$ & $170(41.3)$ \\
\hline \multicolumn{3}{|l|}{ Medium of instruction, $n(\%)^{* *}$} \\
\hline Marathi & $194(46.9)$ & $198(47.9)$ \\
\hline Hindi & $105(25.4)$ & $61(14.8)$ \\
\hline Urdu & $84(20.3)$ & $131(31.7)$ \\
\hline English & $31(7.5)$ & $23(5.6)$ \\
\hline Pocket money/week (INR), mean (SD) & $78.5(82.5)$ & $83.6(79.6)$ \\
\hline \multicolumn{3}{|l|}{ Outcome variables at baseline } \\
\hline Tobacco use prevalence, $\mathrm{n}(\%)$ & $67(17.1)$ & $69(17.7)$ \\
\hline Supari use prevalence, n (\%) & $402(97.1)$ & $399(96.1)$ \\
\hline Dual user, n (\%) & $62(15.74)$ & $66(16.88)$ \\
\hline Age of initiation (years), mean (SD) & $11.3(1.7)$ & $11.1(2.1)$ \\
\hline Tobacco knowledge score $0-6$, mean (SD) ** & $4.6(0.9)$ & $4.4(1.1)$ \\
\hline Supari knowledge score $0-2$, mean (SD) ${ }^{* *}$ & $1.7(0.5)$ & $1.5(0.6)$ \\
\hline Refusal score $\mathbf{0 - 3}$, mean (SD) & $2.6(0.8)$ & $2.7(0.7)$ \\
\hline Exposure to tobacco use by friends/family (Y), n (\%) & $347(83.8)$ & $350(84.8)$ \\
\hline Exposure to supari use by friends/family (Y), n (\%) & $364(87.9)$ & $369(89.4)$ \\
\hline Exposure to tobacco or supari use by school staff (Y), n (\%) & $111(56.4)$ & $147(59.3)$ \\
\hline Exposure to advertising in media $(\mathrm{Y}), \mathrm{n}(\%)^{* *}$ & $368(90.4)$ & $343(83.9)$ \\
\hline Knowledge of school policy in action at own school (Y), n (\%) & $336(92.1)$ & $340(95.0)$ \\
\hline
\end{tabular}

**The difference between the control and intervention groups was statistically significant $($ at $\mathrm{p}<0.05)$ when assessed using comparative statistics: t-test and chi-squared for comparing means and proportions, respectively. INR 100 is roughly equal to USD 1.3.

\section{Tobacco and supari use}

We hypothesized that the participation in the LifeFirst program would lead to reduction in the point prevalence of tobacco and supari use. There was no statistically significant difference in these outcome measures across intervention and control groups at the baseline. At the end of the intervention, tobacco use decreased in the intervention group (17.6\% to $11.6 \%$ ) while it increased in the control group ( $17.1 \%$ to $29 \%)$. The difference-indifference estimates, adjusted for relevant covariates, show statistically significant reduction of 17.9 percent points in the intervention group. The supari use decreased in both the groups but it decreased by a greater proportion among the intervention group compared to the control group with an adjusted difference-in-difference estimator suggesting a statistically significant reduction of 38.1 percent points in intervention beyond the reduction in the control group. The intervention not only led to significant reduction in tobacco and supari use but also had protective effects against new uptake of tobacco use: by the end of the study period, 89 supari users in the control group (out of 340) took up tobacco, whereas only 28 in the intervention group (out of 333) did the same (Figure 2).

\section{Knowledge scores}

Both the groups had high knowledge scores for tobacco harms at the baseline with the control group having a marginally greater tobacco knowledge score (4.57 out of 
Table 2. Difference-in-difference analysis for outcome variables

\begin{tabular}{|c|c|c|c|c|c|}
\hline & \begin{tabular}{|} 
Intervention \\
(I)
\end{tabular} & $\begin{array}{l}\text { Control } \\
\text { (C) }\end{array}$ & $\begin{array}{l}\text { Difference } \\
\text { (I-C) }\end{array}$ & $\begin{array}{c}\text { Unadjusted } \\
\text { difference-in- } \\
\text { differences } \\
\text { estimator (SE) }\end{array}$ & $\begin{array}{c}\text { Adjusted } \\
\text { difference-in- } \\
\text { differences } \\
\text { estimator }^{\mathrm{a}} \text { (SE) }\end{array}$ \\
\hline \multicolumn{6}{|c|}{ Point prevalence of tobacco use (SE) } \\
\hline Before (B) & 0.176 & 0.171 & $0.006(0.028)$ & & \\
\hline After (A) & 0.116 & 0.29 & $-0.173(0.027)^{* *}$ & & \\
\hline Difference (A-B) & & & & $-0.179(0.039)^{* *}$ & $-0.175(0.038)^{* *}$ \\
\hline \multicolumn{6}{|c|}{ Point prevalence of supari use (SE) } \\
\hline Before (B) & 0.966 & 0.971 & $-0.005(0.024)$ & & \\
\hline After (A) & 0.232 & 0.618 & $-0.386(0.024)^{* *}$ & & \\
\hline Difference $(A-B)$ & & & & $-0.381(0.034)^{* *}$ & $-0.379(0.034)^{* *}$ \\
\hline \multicolumn{6}{|c|}{$\begin{array}{l}\text { Tobacco knowledge score (SE) } \\
\text { (Range: } 0-6 \text { ) }\end{array}$} \\
\hline Before (B) & 4.375 & 4.572 & $-0.197(0.065)^{* *}$ & & \\
\hline After (A) & 4.978 & 4.778 & $0.200(0.065)^{* *}$ & & \\
\hline Difference (A-B) & & & & $0.398(0.092)^{* *}$ & $0.406(0.093)^{* *}$ \\
\hline \multicolumn{6}{|c|}{ Supari knowledge score (SE) (Range: 0-2) } \\
\hline Before (B) & 1.547 & 1.698 & $-0.151(0.035)^{* *}$ & & \\
\hline After (A) & 1.831 & 1.751 & $0.079(0.035)^{* *}$ & & \\
\hline Difference (A-B) & & & & $0.230(0.049)^{* *}$ & $0.229(0.05)^{* *}$ \\
\hline \multicolumn{6}{|c|}{$\begin{array}{l}\text { Refusal score for tobacco products (SE) } \\
\text { (Range: } 0-3 \text { ) }\end{array}$} \\
\hline Before (B) & 2.712 & 2.599 & $0.113(0.047)^{* *}$ & & \\
\hline After (A) & 2.814 & 2.688 & $0.125(0.047)^{* *}$ & & \\
\hline Difference (A-B) & & & & $0.012(0.067)$ & $0.018(0.068)$ \\
\hline
\end{tabular}

a Adjusted with covariates (sex, age, standard, school medium, pocket money). ${ }^{* *} \mathrm{p}<0.05$.

Figure 2. Change in product-specific usage over study period (in intervention and control groups) CONTROL GROUP INTERVENTION GROUP

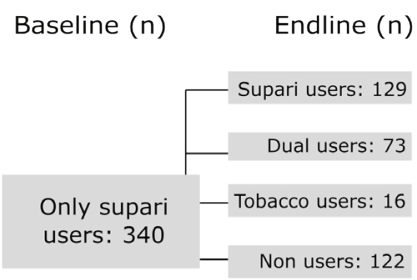
Baseline ( $n$ ) Endline ( $n$ )
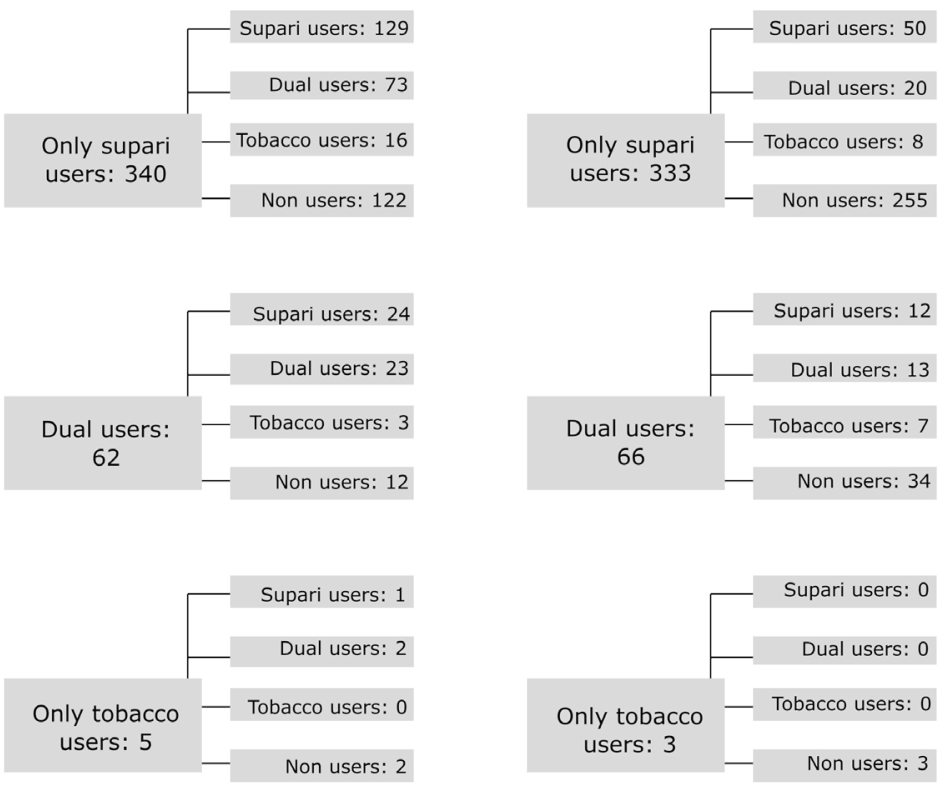
6) compared to the intervention group (4.37 out of 6 ). The same was true for the knowledge score about supari harms. While the knowledge scores improved for both the groups during the intervention period, it improved by a greater percentage among the intervention group compared to the control group; the adjusted difference-in-difference estimator showed a net increase of 0.41 and 0.21 in tobacco and supari knowledge scores among the intervention group. This difference was statistically significant.

\section{Refusal score}

Refusal score assessed the ability of students to refuse offers of uptake of tobacco from their near ones. We found that the refusal score significantly improved in both the groups during the intervention. While the improvement was greater among the intervention group compared to the control group, the net increase in the refusal score among the intervention group was very marginal $(0.23)$ and was statistically insignificant.

\section{DISCUSSION}

Our study shows that the LifeFirst program was successful in reducing tobacco and supari use among students attending the corporation schools in Mumbai. It not only led to reduction in tobacco and supari use but also protected students in the intervention group against new uptake of tobacco. Additionally, it also helped improve knowledge score as well as refusal skills among students. The knowledge of health harms was considerably high to begin with in both the groups. Despite this, students were using some tobacco or supari products. Thus, this implies that only high knowledge of health harms is not sufficient as a solution, and this has to be combined with cessation support to result in behavior change.

We believe our study adds significantly to limited evidence available in India for school-based tobacco control interventions. While there are many school-based tobacco control programs in India, including school-based components of the National Tobacco Control Program, there are only a few focused on behavior modification interventions. And among school-based programs, there is a dearth of published evaluative research on impact of these programs. While not strictly comparable with the LifeFirst program, an evaluation of a multicomponent school-based tobacco control intervention had shown reduction in tobacco use (especially, cigarette and bidi smoking) as well as intention to take up tobacco use in future among 6th and 8th grade students in Delhi and Chennai cities over the twoyear intervention period ${ }^{19}$. Beyond this, we were unable to find other published evidence on school-based interventions in India comparable to the LifeFirst program. This implies not only the potential of school-based interventions for reducing tobacco and supari use among youth, but also the need to do evaluative research around ongoing school-based interventions in India.
The LifeFirst program specifically engaged with students in corporation (local government) schools that generally come from lower socioeconomic class. Youth in general and those coming from lower socioeconomic classes in particular are more vulnerable to tobacco use $\mathrm{e}^{32,33}$. Furthermore, the program's emphasis on offering cessation support for tobacco as well as supari use seems to be responding to a large gap in such support services for students in India. While the number of students using tobacco was limited in the LifeFirst cohort, there was a huge diversity of products that were used by students, including surprise reports of use of e-cigarettes (referred to by students as 'pen-hukka') by many students. It highlights how the tobacco industry targets young people and the relevance of the regulatory measures by governments prohibiting e-cigarettes (electronic nicotine delivery devices) in India to protect youth ${ }^{15}$. The program also shows the importance of addressing a high prevalence of supari use among youth. Our findings show that during the course of the study many students quit supari use but began using tobacco. This further strengthens the belief that detailed studies are warranted to establish if supari is a gateway to tobacco use among adolescents ${ }^{9,10,34}$.

\section{Strengths and limitations}

While a robust experimental design with high response rate and the use of difference-in-difference analysis reflects the strength of our study, there are certain limitations too. We ended up with a limited number of tobacco users (about 17\%) in our sample population, with the majority being supari users. While we still find statistically significant change in our outcome variable, the small number limits the possibility for further analysis segregated by gender and age - something we would have liked to do. We did not use biomarkers owing to the study context and study population and relied on self-report to assess tobacco and supari use status by students. Selfreported responses may be subject to social desirability bias, but as we use the same measures (assessed through the same questionnaires) over time and across groups, we believe that a comparative analysis would still provide a reliable measure of change over time. Self-reported current tobacco use among adolescents has been found to be a valid and stable indicator that can be suitable for public health research ${ }^{35}$. Finally, our sampling (and the explicit LifeFirst program strategy) presents a self-selection bias where tobacco and supari users willingly register for the program. Hence, our encouraging results should be read in the context of student users who are willing to quit tobacco and/or supari and cannot be generalized to all student users.

\section{Implications}

Our work with school-based interventions implemented in schools across Mumbai suggests that structured, long-term tobacco and supari cessation interventions within school settings could help young people quit their tobacco use and 
prevent initiation of tobacco use.

We argue that there is a need for more such interventions as part of a multi-strategy approach to reducing tobacco and supari use and a need for systematic evaluation of these interventions.

\section{CONCLUSIONS}

The LifeFirst school-based tobacco and supari cessation program appears to be effective in helping students using tobacco and supari to stop their habit. For future initiatives, we believe adding a component of cessation (using content and activities suited for adolescents) to interventions aimed at improving knowledge of students will augment the impact of such programs.

\section{REFERENCES}

1. Drope J, Schluger N, Cahn Z, et al. The Tobacco Atlas. 2018. Accessed January 18, 2021. https://tobaccoatlas.org/wpcontent/uploads/2018/03/TobaccoAtlas_6thEdition_LoRes_ Rev0318.pdf

2. Jha P, Jacob B, Gajalakshmi V, et al. A nationally representative case-control study of smoking and death in India. N Engl J Med. 2008;358(11):1137-1147. doi:10.1056/NEJMsa0707719

3. Sinha DN, Palipudi KM, Gupta PC, et al. Smokeless tobacco use: a meta-analysis of risk and attributable mortality estimates for India. Indian J Cancer. 2014;51(Suppl 1):S73-S77. doi:10.4103/0019-509X.147477

4. Global Adult Tobacco Survey, Fact Sheet India: 20092010. Ministry of Health and Family Welfare, Government of India, International Institute for Population Sciences. Accessed January 18, 2021. https://www.who.int/tobacco/ surveillance/india_fact_sheet.pdf?ua $=1$

5. Ministry of Health and Family Welfare, Government of India, World Health Organization, Regional Office for South-East Asia. Global Youth Tobacco Survey 2009. 2009. Accessed January 18, 2021. https://www.who.int/fctc/reporting/ Annexoneindia.pdf

6. Tata Institute of Social Sciences, Mumbai and Ministry of Health and Family Welfare, Government of India. Global Adult Tobacco Survey GATS 2 India 2016-17. 2018. Accessed January 18, 2021. https://ntcp.nhp.gov.in/assets/document/ surveys-reports-publications/Global-Adult-Tobacco-SurveySecond-Round-India-2016-2017.pdf

7. Chandrupatla SG, Tavares M, Natto ZS. Tobacco Use and Effects of Professional Advice on Smoking Cessation among Youth in India. Asian Pac J Cancer Prev. 2017;18(7):18611867. doi:10.22034/APJCP.2017.18.7.1861

8. Garg A, Chaturvedi P, Gupta PC. A review of the systemic adverse effects of areca nut or betel nut. Indian J Med Paediatr Oncol. 2014;35(1):3-9. doi:10.4103/0971-5851.133702

9. Chatterjee N, Todankar P, Mandal G, et al. Factors Associated with Tobacco Use in Students Attending Local Government Schools in Mumbai, India. Asian Pac J Cancer Prev. 2016;17(12):5075-5080. doi:10.22034/APJCP.2016.17.12.5075 10. Rose ML, Chadha D, Bhutia TD. Smokeless tobacco use and perceptions of risk among students in Mumbai municipal schools. Indian J Cancer. 2016;53(2):322-324. doi:10.4103/0019-509X.197720

11.Campaign for Tobacco-Free Kids. India Tiny Targets Report: Tobacco Advertisement, Sales, Product Displays, and Purchase Incentives around Primary and Secondary Schools. 2018. Accessed January 18, 2021. https://www.takeapart. org/wp-content/uploads/2019/01/IndiaTinyTargets2019. pdf

12. Bhojani U. Youth and Tobacco Use: A Monograph on Perceptions, Practices \& Policies. Institute of Public Health; 2013. Accessed January 18, 2021. https://www.researchgate. net/profile/Upendra-Bhojani/publication/262413900_ Youth_and_Tobacco_Use_A_Monograph_on_Perceptions_ Practices_Policies/links/00463537a4f2af3a7c000000/ Youth-and-Tobacco-Use-A-Monograph-on-PerceptionsPractices-Policies.pdf

13. Mishu MP, Siddiqui F, Shukla R, Kanaan M, Dogar O, Siddiqi K. Predictors of Cigarette Smoking, Smokeless Tobacco Consumption, and Use of both forms in Adolescents in South Asia: A Secondary Analysis of the Global Youth Tobacco Surveys. Nicotine Tob Res. 2020;1-10. doi:10.1093/ntr/ntaa202

14. World Health Organization. Healthy Settings: Types of Healthy Settings. Accessed January 18, 2021. https://www. who.int/healthy_settings/types/en/

15. Ministry of Law and Justice. The Prohibition of Electronic Cigarettes (Production, Manufacture, Import, Export, Transport, Sale, Distribution, Storage and Advertisement) Ordinance, 2019. September 18, 2019. Accessed January 18, 2021. https://prsindia.org/files/bills_acts/bills_parliament/ Prohibition \%20 of $\% 20$ Electronic $\% 20$ Cigarettes $\% 20$ Ordinance,\%202019.pdf

16. Ministry of Health and Family Welfare, Government of India, National Health Mission. National Tobacco Control Programme (NTCP). Accessed January 18, 2021. https:// nhm.gov.in /index1.php?lang=1\&level $=2 \&$ sublinkid $=$ 1052 \&lid=607 \# : : text=Government $\% 20$ of $\% 20$ India $\% 20$ launched $\% 20$ the,ensure $\% 20$ effective $\% 20$ implementation $\% 20$ of $\% 20$ the

17. World Health Organization. WHO report on the global tobacco epidemic 2019: Offer help to quit tobacco use. July 25, 2019. Accessed January 18, 2021. https://apps.who.int/ iris/rest/bitstreams/1239531/retrieve

18. Sorensen G, Pednekar MS, Sinha DN, et al. Effects of a tobacco control intervention for teachers in India: results of the Bihar school teachers study. Am J Public Health. 2013;103(11):2035-2040. doi:10.2105/AJPH.2013.301303

19. Perry CL, Stigler MH, Arora M, Reddy KS. Preventing tobacco use among young people in India: Project MYTRI. Am J Public Health. 2009;99(5):899-906. doi:10.2105/AJPH.2008.145433

20. Fanshawe TR, Halliwell W, Lindson N, Aveyard P, LivingstoneBanks J, Hartmann-Boyce J. Tobacco cessation interventions for young people. Cochrane Database Syst Rev. 2017;(11):CD003289. doi:10.1002/14651858.CD003289.pub6 
21. Stead LF, Carroll AJ, Lancaster T. Group behaviour therapy programmes for smoking cessation. Cochrane Database Syst Rev. 2017;(3):CD001007. doi:10.1002/14651858.CD001007.pub3

22. Gupta PC, Ray CS, Papke RL, et al. Perspectives on areca nut with some global implications: Symposium report. Translational research in oral oncology. 2018;3:1-8. doi:10.1177/2057178X18814068

23. Des Jarlais DC, Lyles C, Crepaz N; TREND Group. Improving the reporting quality of nonrandomized evaluations of behavioral and public health interventions: the TREND statement. Am J Public Health. 2004;94(3):361-366. doi:10.2105/ajph.94.3.361

24. Wing C, Simon K, Bello-Gomez RA. Designing Difference in Difference Studies: Best Practices for Public Health Policy Research. Annu Rev Public Health. 2018;39:453-469. doi:10.1146/annurev-publhealth-040617-013507

25. Municipal Corporation of Greater Mumbai. Chapter 2: The particulars of organization, functions \& duties of the Department. Municipal Corporation of Greater Mumbai, Department Manuals. Accessed January 18, 2021. https:// portal.mcgm.gov.in/irj/go/km/docs/documents/MCGM\%20 Department\%20List/Education\%200fficer/RTI\%20 Manuals/Education_Officer_RTI_E02.pdf

26. PRAJA. White Paper: State of municipal education in Mumbai. December 2017. Accessed January 18, 2021. https://www. praja.org/praja_docs/praja_downloads/State $\% 20$ of $\% 20$ Municipal\%20Education\%20in\%20Mumbai.pdf

27. Global Youth Tobacco Survey Collaborative Group. Global Youth Tobacco Survey (GYTS): Core Questionnaire with Optional Questions, Version 1.2. November 2014. Accessed January 18, 2021. https://www.paho.org/hq/ dmdocuments/2017/1-GYTS-CoreQuestionnairewithOptio nalQuestions-v1.2-Nov2014.pdf

28. Bhojani UM, Elias MA, Devadasan N. Adolescents' perceptions about smokers in Karnataka, India. BMC Public Health. 2011;11:563. doi:10.1186/1471-2458-11-563

29. EpiData Analysis. Version 4.6.2.0. EpiData Association; 2014.

\section{ACKNOWLEDGMENTS}

We gratefully acknowledge the cooperation of the study participants and the support of the school principals, staff and LifeFirst intervention team - Sadashiv Hattarki, Asha Kamble Shridhar Mulam, Nitesh Agre and Vishal Sodaye.

\section{CONFLICTS OF INTEREST}

HG and GM are affiliated with the Narotam Sekhsaria Foundation and Salaam Bombay Foundation, respectively, and are part of the team that implements the LifeFirst school program. They did not participate in direct data collection and analysis. Institute of Public Health, Bengaluru, with which UB, AV and PH are affiliated, received fees from the Narotam Sekhsaria Foundation to conduct the study.

\section{FUNDING}

The study was funded by the Narotam Sekhsaria Foundation, in part through subsidizing the foundation-led staff and activities contributing to the study, and in part through a consultancy fee to the Institute of Public Health, Bengaluru. A part of the time of UB and AV (September 2018 onward) was supported through the DBT/Wellcome Trust India
Accessed January 18, 2021. http://www.epidata.dk

30. Stata 15. Version 15. Stata Corp; 2017. Accessed January 18, 2021. http://www.stata.com/

31. Bhojani U, Varma A, Hebbar P, Mandal G, Gupte HA. LifeFirst School-based Tobacco and Supari Cessation Intervention Evaluation Database. Figshare; 2020. doi:10.6084/m9.figshare.12558773.v1

32. Mathur C, Stigler MH, Perry CL, Arora M, Reddy KS. Differences in prevalence of tobacco use among Indian urban youth: the role of socioeconomic status. Nicotine Tob Res. 2008;10(1):109-116. doi:10.1080/14622200701767779

33. Nagrath D, Mathur MR, Gupta R, Zodpey SP. Sociodemographic and socioeconomic differences in tobacco use prevalence among Indian youth. Prev Med Rep. 2019;14:100832. doi:10.1016/j.pmedr.2019.100832

34. Chandra PS, Mulla U. Areca nut: the hidden Indian 'gateway' to future tobacco use and oral cancers among youth. Indian J Med Sci. 2007;61(6):319-321. doi:10.4103/0019-5359.32675

35. Valladolid-López Mdel C, Barrientos-Gutiérrez T, ReynalesShigematsu LM, et al. Evaluating the validity of selfreported smoking in Mexican adolescents. BMJ Open. 2015;5(10):e007485. doi:10.1136/bmjopen-2014-007485

Alliance fellowship (IA/CPHI/17/1/503346) awarded to UB and a part of the time of PH was supported through the DBT/Wellcome Trust India Alliance fellowship (IA/CPHE/17/1/503338) awarded to PH.

\section{ETHICAL APPROVAL AND INFORMED CONSENT}

The study received a formal approval from the Joint Ethics Committee of the Narotam Sekhsaria Foundation and the Salaam Bombay Foundation. The LifeFirst sought approvals from relevant authorities from state and school administrations before engaging with these schools. We obtained written informed consent from parents, followed by informed verbal assent from students before administering the questionnaire. Participation in the program was voluntary.

\section{DATA AVAILABILITY}

All data underlying the results are available as part of the article and the dataset is published on publicly accessible research and data repository, Figshare $^{31}$.

\section{PROVENANCE AND PEER REVIEW}

Not commissioned; externally peer reviewed. 